\title{
Dim galaxies and outer halos of galaxies missed by 2MASS? The near-infrared luminosity function and density
}

\author{
S. Andreon^ \\ INAF - Osservatorio Astronomico di Capodimonte, Naples, Italy \\ Received 24 August 2001 / Accepted 6 November 2001

\begin{abstract}
By using high-resolution and deep $K_{\mathrm{s}}$ band observations of early-type galaxies of the nearby Universe and of a cluster at $z=0.3$ we show that the two luminosity functions (LFs) of the local universe derived from 2MASS data miss a fair fraction of the flux of the galaxies (more than 20 to $30 \%$ ) and a whole population of galaxies of central brightness fainter than the isophote used for detection, but bright enough to be included in the published LFs. In particular, the fraction of lost flux increases as the galaxy surface brightness become fainter. Therefore, the so far derived LF slopes and characteristic luminosity as well as luminosity density are underestimated. Other published near-infrared LFs miss flux in general, including the LF of the distant field computed in a 3 arcsec aperture.
\end{abstract}

Key words. galaxies: evolution - galaxies: clusters: general - X-rays: general

\section{Introduction}

The luminosity function (LF) is the benchmark against which theories of galaxy formation and evolution in a variety of cosmological models can be tested. Therefore, the LF is fundamental to observational cosmology and theory of galaxy formation. In particular, the near infrared LF is a good tracer of evolved stellar populations and hence of the total stellar content of galaxies, much better than optical LFs affected by dust extinction and young stellar populations. Near-infrared luminosities are more directly related to stellar mass, constraining both the history of the star formation and the galaxy formation models (see, e.g., Cole et al. 2000 and references therein)

The luminosity density, which is the integral of the luminosity weighted by the LF, is an important input to estimates of star formation history of the universe, its chemical evolution and of the extragalactic background light.

The recent release of near-infrared imaging data of a large fraction of the sky by 2MASS prompted two groups to derive the near-infrared of the local universe: Kochanek et al. (2001), by using literature or new redshift data, computed the LF of a very nearby sample of galaxies spread over a large sky area, while Cole et al. (2001) coupled near-infrared data to the $2 \mathrm{dF}$ redshift survey and studied a deeper sample over a smaller area.

\footnotetext{
* e-mail: andreon@brera.mi.astro.it
}

The Kochanek et al. (2001) LF has been computed by adopting isophotal magnitudes at the $20.0 \mathrm{mag} \operatorname{arcsec}^{-2}$, $K_{20}$, of a large sample $(\sim 4000)$ of galaxies selected to have $K_{20}<11.25 \mathrm{mag}$, extracted from the 2MASS extended object catalog (Jarrett et al. 2000). A flat LF $(\alpha \sim-0.9)$ has been found both for early-type galaxies and for latetype galaxies separately, and $(\alpha \sim-1)$ for the whole sample. Cole et al. (2001) LF also uses 2MASS data and found similar results in terms of slope $(\alpha=-1.0)$. They used a Kron-like $J$ magnitude and $J-K$ aperture color for measuring the $K$ flux of objects. Both works based on 2MASS photometry found flat slopes.

The found slope is significatively shallower than the one derived by Andreon \& Pelló (2000) for the Coma cluster LF in the $H$ band $(\alpha \sim-1.3)$, and from that derived for the cluster AC 118 (Abell 2744) at $z=0.3$ in the $K_{\mathrm{s}}$ band $(\alpha \sim-1.3)$, for a sample of galaxies outside the cluster core where cluster-related effect should be low.

Furthermore, Wright (2001) notes that the luminosity density based on 2MASS data is between 1.4 and 2.5 times fainter than the one expected by assuming the SLOAN luminosity densities (derived from Blanton et al. 2001 LFs) and a typical spiral spectrum. A redder, elliptical-like or dusty, spectrum would only increase this disagreement.

In this paper we check whether the flat slope and the low luminosity density derived from 2MASS data are affected by flux (and galaxy) losses because of the shallower data set used ( $3 \mathrm{~s}$ exposures). We also show that other LFs 
computed by using aperture magnitudes are skewed with respect to the true one.

In this paper we assume $H_{0}=50 \mathrm{~km} \mathrm{~s}^{-1} \mathrm{Mpc}^{-1}$ and $q_{0}=0.5$, but the choice of the cosmology is largely irrelevant.

\section{LF dependence on the choice of the magnitude adopted}

\subsection{The data}

In order to understand if a significant fraction of the galaxy flux is missed in the near-infrared, we consider two comparison datasets: Pahre (1999) galaxies and AC 118 galaxies. Pahre galaxies are in the nearby universe, are normal and common early-type galaxies on the Fundamental Plane and have been observed by pointed observations deeper and of higher resolution than the 2MASS survey data. Pahre (1999) lists effective radius $r_{\mathrm{e}}$ and brightness $\mu_{\mathrm{e}}{ }^{1}$ and total magnitude for his galaxies. All types of magnitude (isophotal, aperture, Kron, etc.) can be easily computed, assuming that the galaxy profile is accurately described by a de Vaucouleurs (1948) law. This assumption is the major limitation of this dataset.

The second dataset is based on high resolution and deep near-infrared images of the cluster AC 118 (Andreon 2001) at $z \sim 0.3$. This dataset is a well controlled one being a volume complete sample, but the rest-frame spatial resolution is much worser than for the Pahre sample because of the large cluster distance. Unlike the Pahre sample, this dataset includes galaxies of all morphological types, even if early-type galaxies are the majority, being a cluster sample. For the images of these galaxies we directly compute several types of magnitudes (isophotal, aperture and Kron-like) from pixel values, thus avoiding the principal assumption done adopting the Pahre sample. In this respect the two samples are complementary.

\subsection{MASS isophotal magnitudes and the Kochanek et al. $L F$}

Most of the Pahre galaxies are in the same Universe volume studied by Kochanek et al. (2001). With respect to Kochanek's et al. sample, the Pahre sample is $1.5 \mathrm{mag}$ deeper, but we found that when culled at the same apparent magnitude shows the same absolute magnitude distribution. Therefore, the Pahre sample does not grossly undersample galaxies of any absolute magnitude present in Kochanek's et al. sample although it is not a well controlled sample, as the latter. By numerally integrating the galaxy profile down to the 20 mag $\operatorname{arcsec}^{-2}$ isophote we can compute the fraction of the detected flux by 2MASS data. Figure 1 shows the fraction of flux within the $20 \mathrm{mag} \operatorname{arcsec}^{-2}$ isophote as a function of the object magnitude. The missed flux could be as large as $70 \%$. For

\footnotetext{
1 Pahre (1999) lists mean surface brightness within $r_{\mathrm{e}}$, which is equal to $\mu_{\mathrm{e}}-1.39$ adopting a de Vaucouleurs (1948) law.
}

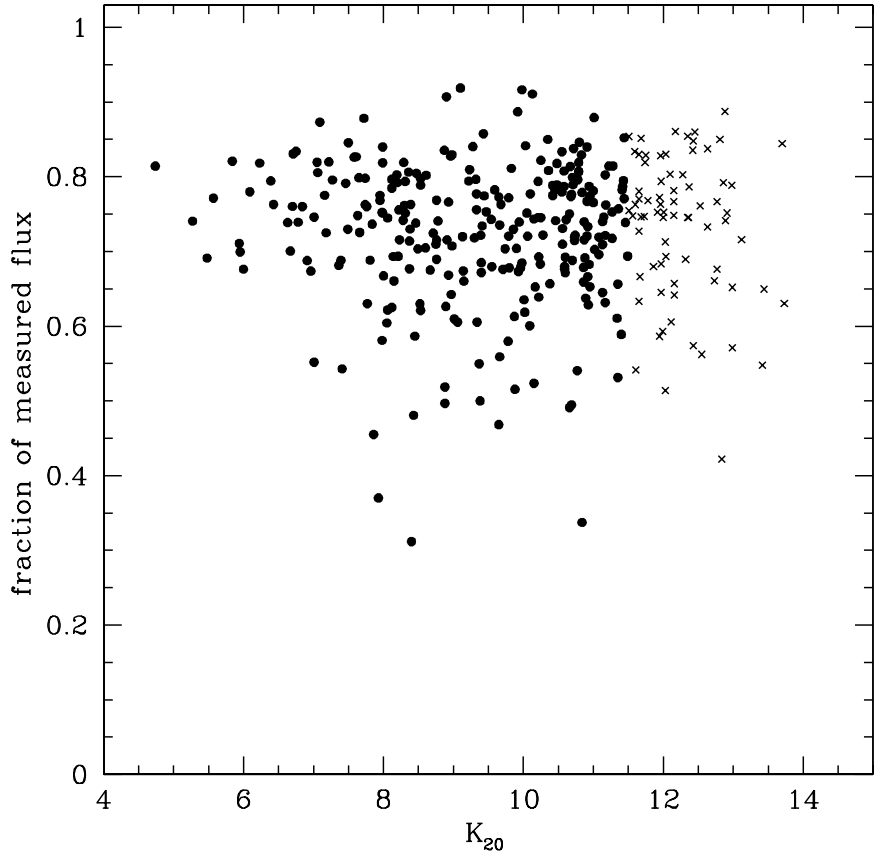

Fig. 1. Fraction of the flux inside the $20 \mathrm{mag} \operatorname{arcsec}^{-2}$ isophote vs. apparent magnitudes for galaxies in Pahre (1999). Filled points are for galaxies with $K<11.5 \mathrm{mag}$, which mimics the Kochanek et al. $K_{20}<11.25$ mag selection (the slightly difference in limiting mag take into account the fact that the isophotal mag miss some flux). Crosses are for fainter galaxies. The isophotal magnitude losts at least $20 \%$ of the flux. This and the following plots (with the exceptions of Figs. 3 and 6) consider about 300 galaxies.

about $60 \%$ of the sample it is larger than $30 \%$ and larger than $15 \%$ for more than $97 \%$ of the sample, much larger that the value claimed by Kochanek et al. (10 to $20 \%$ for most of the galaxies). Figure 2 shows that the fraction of flux lost is mainly a function of the galaxy surface brightness alone. This holds because the dependence on the effective radius of the fraction of missed flux can be factorized and reduced in the plotted ratio. Galaxies, even bright $(K \sim 10$ ) ones, have effective brightness not too different from the $20 \mathrm{mag} \operatorname{arcsec}^{-2}$ detection isophote and a significant part of their flux is lost below the detection isophote. In particular, $50 \%$ of the flux is lost, by definition, when $\mu_{\mathrm{e}}=20 \mathrm{mag} \operatorname{arcsec}^{-2}$, i.e. when the detection and effective isophotes are equal.

The absolute magnitude dependence of the missed flux is negligible for the Pahre sample. However, this dependence cannot be definitively ruled out because the Pahre sample is not complete and the dependence is expected via the correlation between absolute magnitude and surface brightness of galaxies (faint galaxies tend to be of low surface brightness, Andreon \& Cuillandre 2002).

To summarize, the Pahre sample shows that 2MASS isophotal mag lost a significant part of the galaxy flux, larger than the claimed 10 to $20 \%$. Therefore, characteristic luminosity, the luminosity density and possibly the slope of the LF are underestimated by adopting this isophotal magnitudes. 


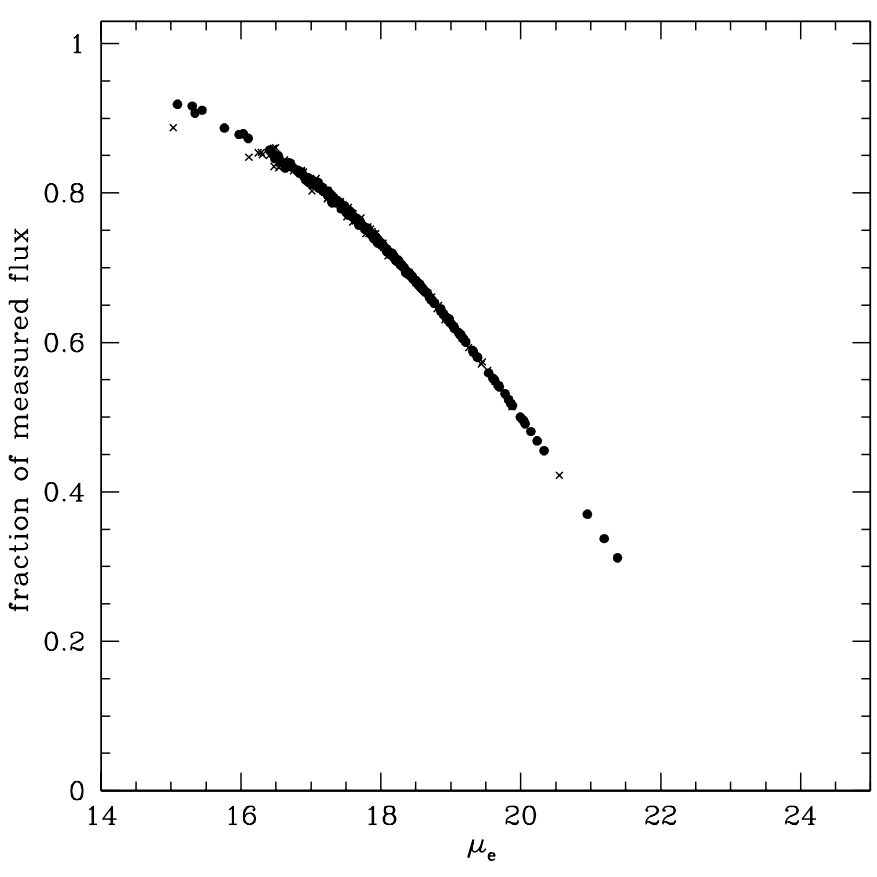

Fig. 2. Fraction of the flux inside the $20 \mathrm{mag} \operatorname{arcsec}^{-2}$ isophote vs. effective surface brightness for galaxies in Pahre (1999). Symbols are as in the previous figure. The reason why the flux is lost is evident: even bright (see previous figure) galaxies have $\mu_{\mathrm{e}}$ few mag brighter than the threshold at which the flux is integrated $\left(20 \mathrm{mag} \operatorname{arcsec}^{-2}\right)$ and therefore the profile is integrated over a small surface brightness range.

Cole et al. (2001) independently confirm that the 2MASS isophotal mag missed a fair fraction of the galaxy flux. They show that the 2MASS isophotal mag misses some 0.1 mag with respect the Kron-like mag listed in the 2MASS database, which in turn misses about 0.15 mag with respect the true Kron mag (because computed on a too small object region), which in turn misses 5 to $10 \%$ of the total flux. Therefore the flux lost by the 2MASS isophotal magnitude is 0.3 to $0.35 \mathrm{mag}$ or, $25 \%$ to $30 \%$ of the galaxy flux, in reasonable agreement with our estimate.

Let us now consider the second dataset. Figure 3 compares our "total" $K_{\mathrm{s}}$ magnitude (that will be defined in the next section) vs. the isophotal magnitude within the $21.5 \mathrm{mag} \operatorname{arcsec}^{-2}$, that correspond to $20.0 \mathrm{mag} \mathrm{arcsec}^{-2}$ isophote in the rest-frame, when cosmological dimming and $\mathrm{k}$-correction are taken into account. The dotted line in Fig. 3 is the bisector line. The restframe 20.0 mag $\operatorname{arcsec}^{-2}$ isophotal magnitude is always fainter than our "total" magnitude, by $0.5 \mathrm{mag}$ on average, which in turn is, of course, fainter than the true total magnitude. Therefore, this plot shows that the isophotal magnitude at the 20.0 mag $\operatorname{arcsec}^{-2}$ misses some $40 \%$ flux from the galaxies, in agreement with the previous estimate based on Pahre data. A second effect could be appreciated from Figure 3 by noting the dearth of galaxies at faint magnitudes. There is almost no galaxy as faint as $M_{K_{\mathrm{s}}}=-22 \mathrm{mag}$ while the cluster LF is flat (at worst, see Andreon 2001) at these magnitudes (and the

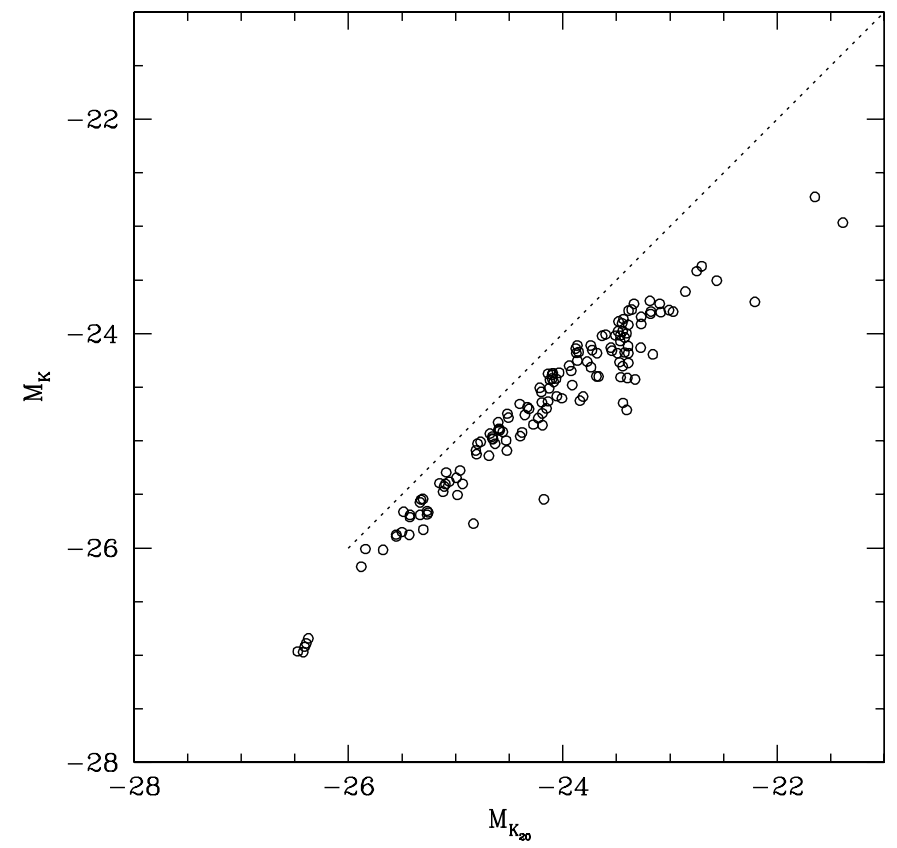

Fig. 3. Our "total" vs. isophotal $\left(\mu=21.5 \mathrm{mag} \operatorname{arcsec}^{-2}\right)$ magnitudes of galaxies in the AC 118 direction. The adopted isophote corresponds to the rest-frame $20 \mathrm{mag} \operatorname{arcsec}^{-2}$ isophote for galaxies at the AC 118 distance. The dotted line is the one-to-one relation. The isophotal mag misses a significant fraction of the galaxy flux, because isophotal mag are much fainter than "total" mag. Note the dearth of faint galaxies (as opposed to the flat of slightly rising cluster LF): their central brightness is so low that they are not detected at all. Therefore, the isophotal magnitude misses also whole galaxies.

background should also contribute with some galaxies). This is a re-stating of the low surface brightness problem: when the central surface brightness drop below the detection isophote the object is undetected. This type of galaxy is bright enough to be included in the local nearinfrared LF but are missed by 2MASS because its surface brightness is too dim. Therefore, for galaxies in our sample, i.e. for an essentially volume complete sample of galaxies in an intermediate redshift cluster, the rest-frame $20 \mathrm{mag} \operatorname{arcsec}^{-2}$ isophote is not a good choice for measuring the LF for two reasons: because of the large fraction of missed flux, and because of the numerous missed galaxies at whole. Both effects produce flat (and faint) LFs and low luminosity densities.

Figure 4 shows that our "total" magnitude is not bad: for most of the Pahre (1999) sample the magnitude within 2.5 Kron radii (Kron 1980) misses an approximately constant $10 \%$ of the galaxy flux. In this specific calculation we take into account that the flux used to compute the second moment of the light distribution (the Kron $r_{1}$ radius) is actually integrated over $12^{2}$ times (Bertin 2001, private communication) the detection area by SEx (Bertin \& Arnout 1996) $)^{2}$. For faint objects $\left(M_{\mathrm{K}} \gtrsim-22 \mathrm{mag}\right)$ we adopted an aperture magnitude, but the aperture is large

\footnotetext{
${ }^{2}$ But only in the detection area for 2MASS objects (Jarrett 2001, private communication cited in Cole et al. 2001).
} 


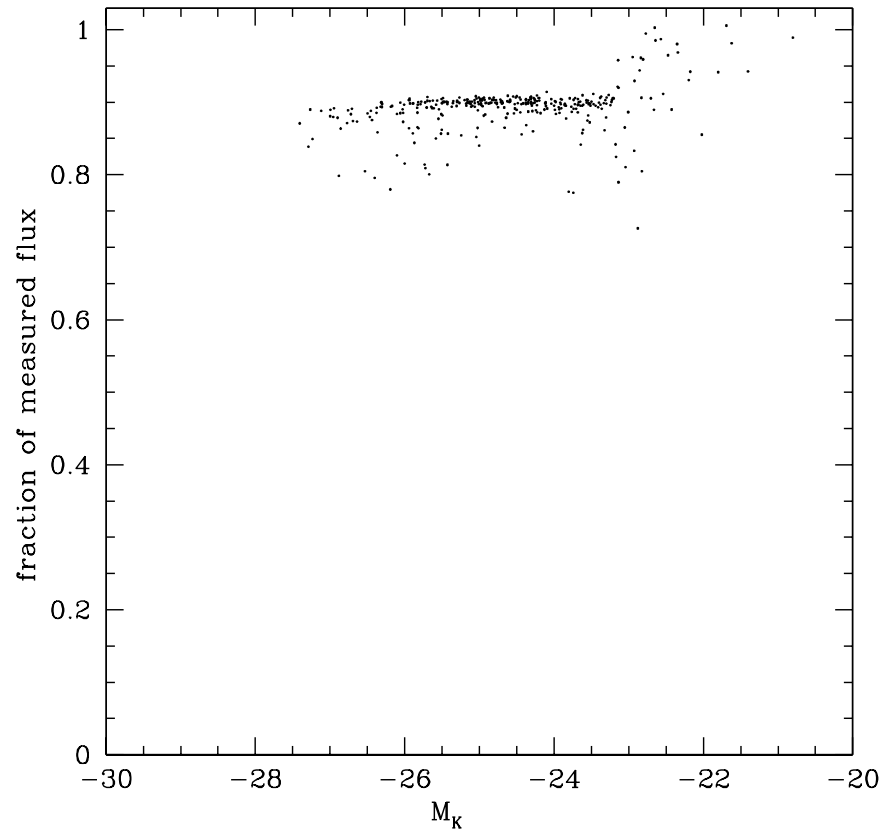

Fig. 4. Fraction of the flux within our "total" magnitude vs. absolute magnitude for the Pahre (1999) sample.

enough to include most of the flux. The ultimate reason for our "total" mag being a successful measure of the galaxy total flux is that the Kron radius is extremely well correlated with the effective radius and the ratio of the two radii is independent of $\mu_{\mathrm{e}}$ and almost constant. Therefore, the galaxy flux is integrated within an almost fixed number of effective radii, which contains an almost constant fraction of the total flux, for a fixed surface brightness profile shape. Outlyer points in Fig. 4 turn out to be galaxies with a so large effective radius that the computed Kron radius is underestimated from the object portion considered by the detection software, much like the usual situation for 2MASS Kron-like magnitudes.

\subsection{MASS Kron-like magnitudes and Cole et al. LF}

By using the Pahre data we can repeat the same previous exercises for 2MASS Kron-like magnitudes used for computing the $2 \mathrm{dF}$ LF (Cole et al. 2001). These mags are measured within 2.5 times the first moment of the light distribution of the pixels brighter than the detection threshold in $J$ (21.7 mag, Jarrett et al. 1999, cited in Cole et al. $2001^{3}$ ) minus the $J-K$ color (computed on a smaller galaxy area). This particular choice allows the $2 \mathrm{dF}$ team to integrate a larger fraction of the galaxy flux (than adopting the Kron $K$ mag), under the hypothesis of minor color gradients between the $J$ and $K$ bands. Again, mimicking the integration of the galaxy flux and assuming the observed average color $J-K=1.1 \mathrm{mag}$ (Cole et al. 2001), we can easily compute the fraction of the flux missed by the magnitudes adopted by the $2 \mathrm{dF}$

\footnotetext{
${ }^{3}$ See also http://spider.ipac.caltech.edu/staff/ jarrett/2mass/repeats/kron.html
}

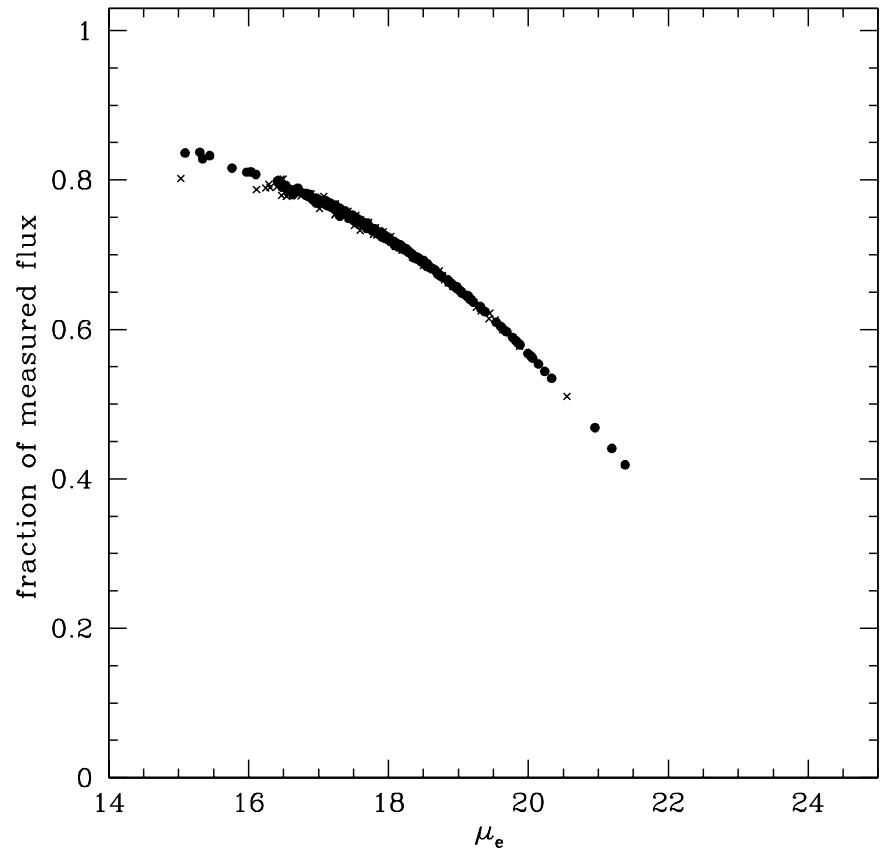

Fig. 5. Fraction of the flux inside the $K$ mag adopted for the $2 \mathrm{dF}$ near-infrared luminosity. Note the similarity with respect to Fig. 2, except for a milder surface brightness dependence.

team (Fig. 5). Unlike SEx, 2MASS computes the moments of the light distribution on a often tiny fraction of the galaxy profile, giving an under-estimation of them and, by consequence, of the 2MASS Kron-like magnitude. Due to the under-estimation of the true Kron radius (even if measured in the deeper $J$ band), the flux of galaxies are underestimated by 20 to $50 \%$, and $0.35 \mathrm{mag}$ on average for the Pahre sample ${ }^{4}$. This result is a bit larger than the value quoted by Cole et al. (2001): they found that the 2MASS Kron-like mag misses 0.06 mag with respect to the Loveday (2000) Kron mag (for a sample of common objects), which in turn misses $10 \%$ (0.1 mag) of the total flux, as is claimed by Cole et al. (2001) and checked by us. Therefore, according to Cole et al. (2001), their mag misses a total of $0.16 \mathrm{mag}$ (for Pahre galaxies, vs. our estimate of $0.35 \mathrm{mag}$ ).

Judging from their Fig. 15, the Cole et al. (2001) estimate of the lost flux is low: their measured LF is still shallower and fainter than expected from the SDSS $z^{*} \mathrm{LF}$ (Blanton et al. 2001) converted in $K$. It is fainter because their correction for missing flux is underestimated. By correcting the Cole et al. (2001) LF by a further $0.2 \mathrm{mag}$ (our 0.35 mag minus 0.16 mag already corrected for), the SDSS and Cole et al. LFs are in reasonable agreement at the bright end and the two LFs have very similar amplitudes $\left(\phi^{*}\right)$ at $M^{*}$. At the faint end, the Cole et al. LF is shallower because galaxies of low surface brightness are

\footnotetext{
${ }^{4}$ A revision of the Kron photometry is planned for the final reprocessing of the 2MASS data, and therefore our criticism likely concerns exclusively Kron magnitudes in the 2MASS incremental releases.
} 

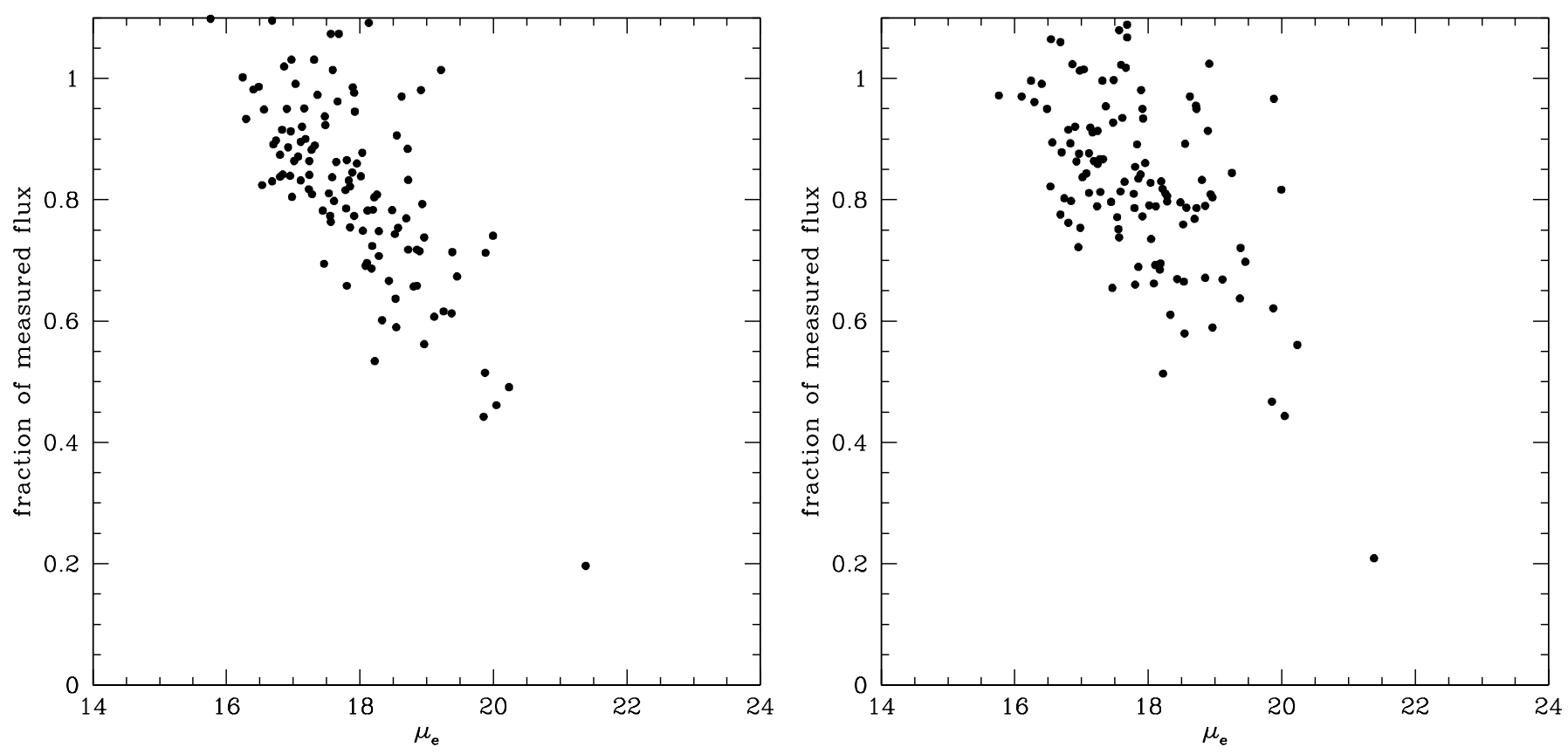

Fig. 6. Fraction of flux lost vs. isophotal effective brightness for galaxies both in the Pahre and 2MASS samples. Left panel: isophotal 2MASS magnitude used by Kochanek et al.; right panel: hybrid Kron magnitude used by $2 \mathrm{dF}$. A few outliers have brighter isophotal and Kron magnitudes than total one, possibly due to nearby objects not perfectly handled by the 2MASS pipeline.

missed because they are too dim, and are not listed in the 2MASS catalog.

\subsection{Further checks}

Since some of the Pahre galaxies are listed in the 2MASS database, it is quite easy to directly measure the fraction of flux lost, because it is given by the difference between the total magnitude, listed in Pahre's paper as measured from the growth curve technic, and 2MASS mags listed in the 2MASS catalog.

Pahre galaxies are identified by name (about half of them are NGC/IC galaxies), while 2MASS galaxies by coordinates. From the Pahre list of 340 galaxies we were able to get sky coordinates from NED for 327 of them. Then, we looked for sources, within a 5 arcsec radius circle centered on NED coordinates, in the second 2MASS incremental release catalog. Out of 327 objects, 122 of them (37\%) are listed in the 2MASS catalog.

The left panel of Fig. 6 shows the fraction of flux lost by the isophotal magnitude adopted by Kochanek et al., $K_{20}$, as a function of the effective surface brightness of the galaxy. A clear trend is present, in agreement with what is found in Sect. 2.2: the missed flux is larger for lower surface brightness galaxies, and could be as large as $50 \%$ when $\mu_{\mathrm{e}}=20 \mathrm{mag} \operatorname{arcsec}^{-2}$, as it should be when the detection and effective isophotes are equal. On average, $K_{20}$ losts $0.2 \mathrm{mag}$ for galaxies listed both in the Pahre sample and in 2MASS, but, of course, the average depends on the $\mu_{\mathrm{e}}$ distribution.
The right panel of Fig. 6 shows the fraction of lost flux by the Kron-like magnitude adopted by $2 \mathrm{dF}$. The result is quite similar to that found for isophotal magnitudes, and it is in agreement with what found in Sect. 2.3: a similar trend for increasing missing flux when effective brightness become fainter is present, and, on average $0.2 \mathrm{mag}$ are lost for galaxies listed both in the Pahre sample and 2MASS.

We stress that we are talking about bright, famous and rare galaxies: these galaxies have on average $K \sim$ -24.7 mag. Galaxies fainter by four, or five, magnitudes are included in the Kochanek et al. and in the 2dF LFs. These ordinary galaxies have fainter surface brightnesses because of the correlation between absolute magnitude and surface brightness, as already noted. Therefore, the fraction of missed flux measured by Fig. 6 is underestimated, when an ordinary sample is chosen. A rough estimate of the typical fraction of flux lost for these normal galaxies can be computed as following: the characteristic magnitude is $K^{*} \sim-25 \mathrm{mag}$ (Kochanek et al. and Cole et al.). These galaxies turn out to have typical $\mu_{\mathrm{e}}$ of $17 \mathrm{mag} \operatorname{arcsec}^{-2}$ (with a very large scatter) in the $K$ band for the Pahre sample. In the optical, Sandage \& Perelmuter (1990) shows that galaxies having $M^{*}+4$ have $\mu_{\mathrm{e}}$ two mag fainter than $M^{*}$ galaxies, on average. Assuming negligible color gradient between optical and infrared colors, galaxies having $M^{*}+4$ have $\mu_{\mathrm{e}} \sim 19 \mathrm{mag} \operatorname{arcsec}^{-2}$ (with a large scatter) in the $K$ band. The same result can be found assuming a reasonable color for galaxies, and reading directly the optical $\mu_{\mathrm{e}}$ at $M^{*}+4$ in, say, Sandage \& Perelmuter (1990). At such a brightness the fraction of flux lost by isophotal mag is $40 \%$ (see Fig. 2 or left panel of Fig. 6). The estimate for the Kron 
magnitude adopted by $2 \mathrm{dF}$ is similar (see Fig. 5 or right panel of Fig. 6).

Figure 6 definitively shows that the fraction of missed flux is easily much larger than $15 \%$, that it is correlated to $\mu_{\mathrm{e}}$ and that it is already large for high surface brightness galaxies. For ordinary galaxies the fraction of lost flux is by necessity larger. Results from Fig. 6 do not assume a surface brightness profile for the galaxies, while Figs. 2 and 5 do, and the agreement between findings drawn from these figures confirms that the assumption of a de Vaucouleurs law is a good one.

The galaxy with the largest fraction of lost flux in Fig. 6 has $K=8.4$ mag.

Since Fig. 6 is produced without almost any work by us (we just paired catalog entries), the probability that a mistake occurred in this plot is very low.

As a final check, we compared our synthetic photometry vs. $2 \mathrm{MASS}$ measures for the $K_{20}$ and Kron-like mags. We found an offset of $\lesssim 0.1 \mathrm{mag}$, with a scatter of $0.1 \mathrm{mag}$, in the sense that 2MASS mag are brighter than ours. While the whole offset is not entirely understood by us, part of it come from known effects described in Jarrett et al. (2001, isophotal contours are uncalibrated at 0.1 to $0.2 \mathrm{mag} \operatorname{arcsec}^{-2}$ ), by some likely operation performed on 2MASS images for isophote regularization (convolution with a kernel) and from the fact of having neglected seeing effect in our computation. We stress that, even without taking into account all these effects, the systematic offset is only a bit larger than the photometric error quoted in the 2MASS catalog (0.08 mag).

Our underestimation of the 2MASS flux (or their overestimation with respect to our modelleing) reduces the $a v$ erage fraction of missed flux lost by 2MASS from about $0.35 \mathrm{mag}$ (claimed in previous sections) to about $0.25 \mathrm{mag}$ for both Kron-like and isophotal mags.

The same $0.1 \mathrm{mag}$ offset helps to reduce the disagreement between the Cole et al. (2001) and our estimate of the fraction of flux lost by their Kron-like magnitude. In fact, when this offset is taken in to account, the two estimates differ by $0.1 \mathrm{mag}$ only.

Nevertheless, Fig. 6 unambiguously shows that the fraction of flux lost depends on $\mu_{\mathrm{e}}$, and could be very large even for galaxies bright and well known enough to have a name. From ordinary galaxies, the same figure shows that the average flux lost is by necessity large.

\subsection{Aperture magnitude and $L F s$ based on them}

The problem described for the local field LF is, in fact, a general one. For AC 118 galaxies (at $z=0.3$ ) our (Andreon 2002) 3 arcsec aperture magnitude (16 Kpc for galaxies at the AC 118 redshift) is not a surrogate for "total magnitude": we checked that a lot of flux is lost by comparing 3 arcsec aperture magnitude to both our surrogate of "total" magnitude (figure not shown) and to total mags of Pahre galaxies (redshifted at the cluster redshift, figure not shown). Therefore, the LF computed

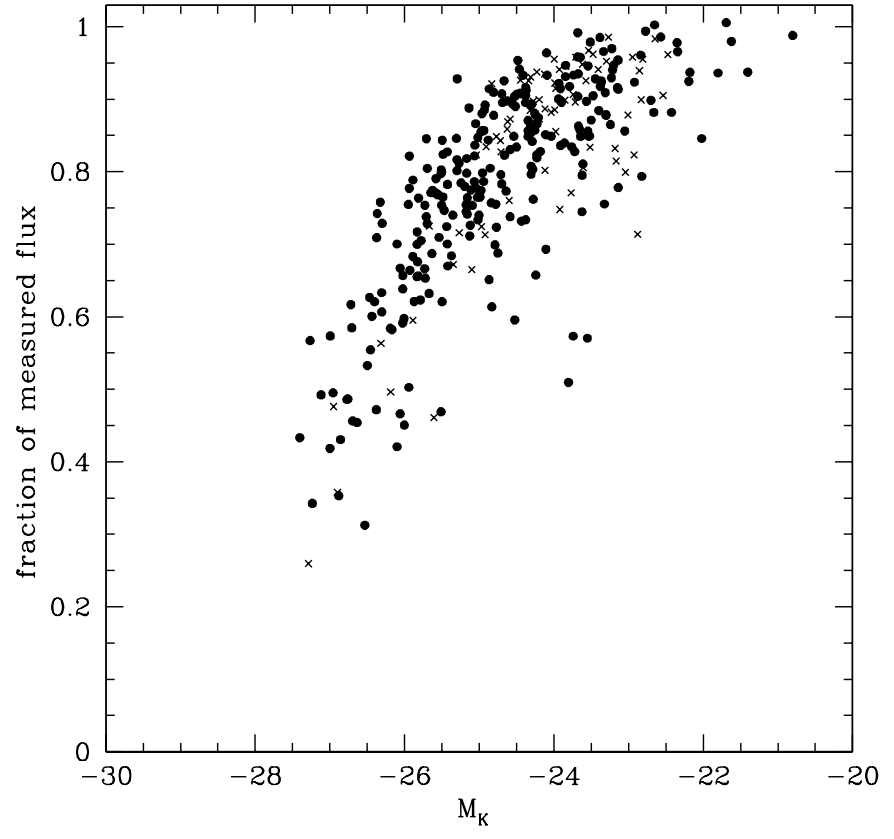

Fig. 7. Fraction of the flux inside the 3 arcsec aperture at $z=0.6$. Symbols as in Fig. 1. The same plot for galaxies at $z=0.3$ is qualitatively similar, except for a larger missing flux, by 0.1 , for all but the faintest galaxies.

by using this aperture is skewed with respect to the true one. Of course, the same holds for the field LF too. Using again the Pahre sample we can compute the fraction of flux missed adopting a 3 arcsec aperture for galaxies at $z=0.6$ (Fig. 7). Even if we recover the known result that, on average, the fraction of missed flux is $0.2 \mathrm{mag}$ (value for which the mag of field galaxies are corrected for), this holds only at intermediate absolute magnitude. For bright galaxies the needed correction is quite large and, most important, the flux lost is large because these galaxies are bright. This flux loss possibly represents a fair fraction of the luminosity density. On the contrary, the applied correction is too large for faint galaxies.

Therefore, the 3 arcsec aperture magnitude, corrected to 6 arcsec aperture by a single average offset (e.g. Cowie et al. 1996), and other similar aperture-corrected magnitudes used for computing the field LF at intermediate redshift, are coarse approximations of the total mag. This approximation holds maybe for galaxies of the same absolute magnitude (possibly of similar brightnesses and scales), but not for galaxies of quite different magnitudes which differ in brightness and scale and lay in different parts of the LF. These approximations are minor problems when errorbars are large because of the smallness of the sample, but when the sample is large, as it is the case of the present-day near-infrared LFs, systematic errors are the largest sources of uncertainty.

\section{Discussion}

By using the Pahre (1999) sample of early-type galaxies lying in the near-universe and on the Fundamental 
Plane and our high resolution and deep near-infrared images of galaxies at intermediate redshift we show that the two recently-determined near-infrared LFs of the nearby Universe based on 2MASS data (Kochanek et al. 2001 and Cole et al. 2001) suffer from flux lost below the detection isophote and by missing galaxies of low surface brightness, but bright enough to be included in the LF.

Three paths have been followed for computing the fraction of missing flux:

- we directly compare published total mag to mag listed in the 2MASS catalog for galaxies in common between Pahre (1999) and the 2MASS second incremental release,

- we simulate the 2MASS magnitude measurement for all the Pahre galaxies by synthetic photometry by assuming a de Vaucouleurs law for their surface brightness profile and we derive the fraction of missed flux, and

- we use actual $K$ images of galaxies in an intermediate redshift cluster.

All three paths give the same results: the fraction of missed flux by isophotal or Kron-like magnitudes is correlated to galaxy effective surface brightness and could be very large for galaxies included in the Kockanek et al. and 2dF LFs. It is larger than quoted in Kochanek et al. and Cole et al. for galaxies in the Pahre list, $(\sim 0.35$ mag from syntetic photometry over the whole sample, $\sim 0.25$ mag from direct comparison of the galaxies in common with the 2MASS database), that are famous and bright enough to have a name. For common galaxies the fraction of the flux lost is larger (Figs. 2, 5 and 6) due to the correlation between absolute magnitude and surface brightness.

The dearth of faint galaxies in Fig. 3, coupled with a flat (at worst) AC 118 LF shows that galaxies of low surface brightness, but bright enough to be included in the Kochanek et al. and Cole et al. LFs, exist but are undetected by 2MASS. This is also the extreme consequence of the previously mentioned effect, when the missed flux is equal to the total flux. Therefore, the slope of the two mentioned LFs is underestimated.

Furthermore, a significant loss of flux has been shown in this paper for the near-infrared LFs based on $3^{\prime \prime}$ aperture mag, as usually adopted for LFs at intermediate redshifts (and also for computing galaxy counts).

The missed flux has, of course, an obvious relevance to the determination of the luminosity density, which, beside the systematic errors shown in this paper, have presently insignificant statistical errors (Cole et al. 2001). Some cosmological consequences of missed flux are reported in Wright (2001).

In general, our considerations of the relevance of the type of magnitude used for the LF computation are confirmed in the optical window by Blanton et al. (2001), that show how much the optical LFs derived thus far, such as the Las Campanas (Lin et al. 1996) and 2dFGRS (Folkes et al. 1999), are skewed with respect the true ones because of the use of isophotal magnitudes. Another similar finding is reported in Garilli et al. (1999): the slope of the LF for a sample of 2200 galaxies is shallower when a $20 \mathrm{Kpc}$ aperture magnitude is used in place of pseudo-total magnitudes.

Summarizing, when the sample is large, of the order of several hundred galaxies, the largest errors on the LF are systematic in nature. A similar conclusion has been suggested by Kochanek et al. (2001) for some LFs split by spectral type (ESP, Zucca et al. 1997; Las Campanas et al. 1996; Bromley et al. 1998; 2dFGRS, Folkes et al. 1999).

Can the true LF be recovered from the skewed one?

First of all, the faint end can hardly be recovered because galaxies missed by 2MASS are not listed at all, and therefore it is unknow how many of them are missing. By using a deeper sample it is possible to determine the absolute magnitude at which the population of galaxies missed by 2 MASS become important and one can limit the LF determination to brighter magnitudes. The completness can be determined, say, by comparing standard and stacked 2MASS observations (for example the cluster Abell 3358 has been scanned 30 times by $2 \mathrm{MASS}$ ), or the 2MASS catalog and published complete deeper near-infrared catalogs (such as Andreon et al. 2000 for the Coma cluster). Our present analysis allows one to measure how much flux is lost per galaxy (detected or not), but not how many galaxies are lost.

At brighter magnitudes, the "total" magnitude could be recovered by using a measure of the galaxy scale, but with some approximation. In fact, the correction from measured mag to total mag depends on the galaxy growth curve, that in turn depends on the scale and brightness of each individual galaxy even under the simplistic assumption that the surface brightness profile of all galaxies is well described by a universal law. Therefore, at least a measure of the galaxy scale is needed to estimate the LF slope and characteristic luminosity. Such a measure is encoded in the 2MASS data products, although it is biased low because the moments of the ligh distribution are computed over often a tiny part of the galaxy surface brightness profile. The final processing of the 2MASS data will use a more elaborate schema for computing Kron radii and magnitudes, as described in http://spider.ipac.caltech.edu/staff/jarrett/ 2mass/repeats/kron.html.

A rough estimate of how much the LF is skewed by adopting magnitudes that are know to miss flux (and whole galaxies) can be guessed by comparing two determinations of the LF of AC 118: the first one is computed by using a 3 arcsec aperture (Andreon 2002) and agrees with both determinations of the local LF, while the second one adopts our surrogate of "total" mag (Andreon 2001): the LF is shallower by 0.3 in $\alpha$ and fainter by $0.9 \mathrm{mag}$ in $M^{*}$ (but be aware that errors on best fit parameters are strongly coupled and therefore other pairs of values give almost equivalent descriptions of the difference).

Acknowledgements. This work is dedicated to my daughters, Chiara and Marta Andreon, the new twin stars born under the sign of Gemini. Discussion with A. Wolter, R. Rampazzo and 
A. Iovino is acknowledged. This work has been performed at the Osservatorio Astronomico di Brera that I thank for hospitality. The author acknowledges the referee for suggesting the comparison presented in Sect. 2.4.

This publication makes use of data products from the Two Micron All Sky Survey, which is a joint project of the University of Massachusetts and the Infrared Processing and Analysis Center/California Institute of Technology, funded by the National Aeronautics and Space Administration and the National Science Foundation This research has made use of the NASA/IPAC Extragalactic Database (NED) which is operated by the Jet Propulsion Laboratory, California Institute of Technology, under contract with the National Aeronautics and Space Administration.

\section{References}

Andreon, S. 2001, ApJ, 547, 623

Andreon, S. 2002, A\&A, in press

Andreon, S., \& Cuillandre, J.-C. 2002, in press

Andreon, S., Pelló, R., Davoust, E., Domínguez, R., \& Poulain, P. 2000, A\&AS, 141, 113

Andreon, S., \& Pelló, R. 2000, A\&A, 353, 479

Bertin, E., \& Arnouts, S. 1996, A\&AS, 117, 393

Blanton, M. R., Dalcanton, J., Eisenstein, D., et al. 2001, AJ, 121,2358
Bromley, B. C., Press, W. H., Lin, H., \& Kirshner, R. P. 1998, ApJ, 505, 25

Cole, S., Lacey, C., Baugh, C., \& Frenk, C. 2000, MNRAS, 319,168

Cole, S., Norberg, P., Baugh, C. M., et al. 2001, MNRAS, 326, 255

Cowie, L. L., Songaila, A., Hu, E. M. \& Cohen, J. G. 1996, AJ, 112,839

de Propris, R., Eisenhardt, P. R., Stanford, S. A., \& Dickinson, M. 1998, ApJ, 503, L45

de Vaucouleurs, G. 1959, Handbuch der Physik, vol. 53 (Berlin: Springer Verlag)

Folkes, S., Rosen, S., Price, I., et al. 1999, MNRAS, 308, 459

Garilli, B., Maccagni, D., \& Andreon, S. 1999, A\&A, 342, 408

Loveday, J. 2000, MNRAS, 312, 557

Kochanek, C., Pahre, M., \& Falco, E. 2001, ApJ, submitted

Kochanek, C., Pahre, M., Falco, E., et al. 2001, ApJ, 560, 566

Kron, R. G. 1980, ApJS, 43, 305

Jarrett, T. H., Chester, T., Cutri, R., et al. 2000, AJ, 119, 2498

Lin, H., Kirshner, R. P., Shectman, S. A., et al. 1996, ApJ, 464,60

Pahre, M. A. 1999, ApJS, 124, 127

Sandage, A., \& Perelmuter, J. 1990, ApJ, 361, 1

Zucca, E., Zanorani. G., Vellotani, G., et al. 1997, A\&A, 326, 477

Wright, E. L. 2001, ApJ, 556, L17 